Homology, Homotopy and Applications, vol.13(2), 2011, pp.165-174

\title{
TOPOLOGICAL INVARIANCE OF THE COMBINATORIAL EULER CHARACTERISTIC OF TAME SPACES
}

\author{
TIBOR BEKE \\ (communicated by Gunnar Carlsson)
}

Abstract

We prove the topological invariance of the combinatorial Euler characteristic with the help of a canonical, topologically defined stratification of tame spaces by locally compact, tame strata.

\section{Introduction}

Intuitively, tame topology studies those subcategories of the category of topological spaces and continuous maps for which an appropriate version of the Hauptvermutung holds: any two triangulations of a tame space will be combinatorially equivalent. (The term tame space is a bit of a misnomer; it is not a topological space as such that deserves to be called tame, but an object of a category of tame spaces and tame maps. In particular, one should talk of tame triangulations of tame spaces and so on. But let us postpone precise definitions till the next section.) The proofs by Hironaka and Lojasiewicz of the triangulability of algebraic and analytic varieties led to the discovery of the paradigmatic tame category: that of affine semi-algebraic spaces and semi-algebraic maps. Recall that a subset of $\mathbb{R}^{n}$ is semi-algebraic if it can be written as a finite boolean combination of subsets of $\mathbb{R}^{n}$ defined by polynomial equalities and inequalities. Such a set need not be locally compact (let alone compact), so the definition of triangulation has to be modified a little: a triangulation of a (semialgebraic) space $X$ is a (semi-algebraic) decomposition of $X$, the pieces of which are (semi-algebraically) homeomorphic to the relative interiors of affine simplices. (See Knebusch and Delfs [DK82] for details.) The usual formula

$$
\mathrm{eu}(X)=\sum_{\alpha \in \operatorname{Simp}(X)}(-1)^{\operatorname{dim}(\alpha)}
$$

the sum running over the set of simplices in the triangulation, defines the combinatorial Euler characteristic. For example, if $X$ is the union of the interior of a 3-simplex with vertices $A B C D$ with the relative interior of the face $A B C$ and the vertex $A$ (this example not being locally compact), then $\mathrm{eu}(X)=1$.

Supported by the NSA under Grant no. H98230-11-1-0172.

Received October 8, 2009, revised June 5, 2011; published on October 31, 2011.

2000 Mathematics Subject Classification: 57N80, 05E45.

Key words and phrases: tame space, Euler characteristic, stratification.

Article available at http://intlpress.com/HHA/v13/n2/a11 and doi:10.4310/HHA.2011.v13.n2.a11

Copyright (C) 2011, International Press. Permission to copy for private use granted. 
To be sure, if $X$ is compact, then $\operatorname{eu}(X)=\sum_{i=0}^{\operatorname{dim}(X)}(-1)^{i} \operatorname{dim} H^{i}(X ; \mathbb{Q})$, which is a homotopy invariant. If $X$ is locally compact, then

$$
\mathrm{eu}(X)=\sum_{i=0}^{\operatorname{dim}(X)}(-1)^{i} \operatorname{dim} H_{c}^{i}(X ; \mathbb{Q}),
$$

the compactly supported cohomological Euler characteristic, which is a proper homotopy invariant. The modest goal of this note is to prove that the combinatorial Euler characteristic is always a homeomorphism invariant. The two facts that give this statement its context are Milnor's disproof of the polyhedral Hauptvermutung and the apparent lack of cohomological interpretation of the combinatorial Euler characteristic of tame spaces that are not locally compact.

The main result will be proven in the setting of o-minimal structures over $\mathbb{R}$. These emerged in the 1990's as the leading axiomatization of Grothendieck's vision of tame topology, and include and extend semi-algebraic topology in several directions.

\section{Tame topology and o-minimal structures}

Definition 2.1. An o-minimal structure $\mathcal{S}$ over the reals is a collection $\left\{\mathcal{S}_{n}\right\}, n \in \mathbb{N}$ such that

(1) for each $n, \mathcal{S}_{n}$ is a boolean algebra of subsets of $\mathbb{R}^{n}$

(2) if $A \in \mathcal{S}_{n}$ and $B \in \mathcal{S}_{m}$, then $A \times B \in \mathcal{S}_{n+m}$

(3) for any $1 \leqslant i<j \leqslant n$, the set $\left\{\left(x_{1}, x_{2}, \ldots, x_{n}\right) \mid x_{i}=x_{j}\right\}$ belongs to $\mathcal{S}_{n}$

(4) if $A \in \mathcal{S}_{n+1}$ and $\pi: \mathbb{R}^{n+1} \rightarrow \mathbb{R}^{n}$ is a projection, then $\pi(A) \in \mathcal{S}_{n}$

(5) singleton sets belong to $\mathcal{S}_{1}$ and the set $\{(x, y) \mid x<y\}$ belongs to $\mathcal{S}_{2}$

(6) if $A \in \mathcal{S}_{1}$, then $A$ is a finite union of intervals (points and half-infinite intervals being also counted among intervals).

This definition is adopted from van den Dries [vdD98]. The axioms are satisfied if $\mathcal{S}_{n}$ is the collection of semi-algebraic subsets of $\mathbb{R}^{n}$; property (4) is then the TarskiSeidenberg theorem. The definition itself, however, is the outgrowth of logicians' examination of the structure of definable sets in certain first-order logical theories. The key property - and reason for the nomenclature "o-minimal", short for "orderminimal" - is (6), stating that no more one-dimensional sets belong to $\mathcal{S}$ than what must be definable in any first-order structure containing a linear order $<$. It is a deep insight that the combinatorial property (6) implies for o-minimal sets the very same tame topological features that, for semi-algebraic sets, are usually derived from commutative algebra.

Semi-linear sets (cf. Schanuel [Sch91]) form an example of an o-minimal structure that is properly included in semi-algebraic sets. Starting from the 90's, remarkable o-minimal structures have been discovered extending semi-algebraic sets. One can think of these as the result of permitting special families of real-analytic functions besides polynomials to serve in the equations and inequalities defining subsets. It is a corollary of a deep result of Rolin-Speissegger-Wilkie [RSW03] that there is no maximal o-minimal structure over the reals. In fact, they give an example of two 
o-minimal structures $\mathcal{S}^{(1)}, \mathcal{S}^{(2)}$ and sets $X_{i}$ belonging to $\mathcal{S}^{(i)}(i=1,2)$ such that no o-minimal structure can contain both $X_{1}$ and $X_{2}$.

van den Dries [vdD98] found a construction of the combinatorial Euler characteristic eu that works for any o-minimal structure and avoids the use of triangulations in favor of the more order-theoretic cylindrical cell decompositions. For semi-algebraic sets, these were introduced by Collins; see Basu-Pollack-Roy [BPR06] Ch. 5. For reference, let us recall their definition after van den Dries [vdD98]. Fix an o-minimal structure $\mathcal{S}$ over the reals. For all topological intents and purposes, the sets belonging to $\mathcal{S}_{n}$ for some $n$ should be called and thought of as tame. (See for example Nicolaescu [Nic10] for this usage.) However, owing to the logical and model-theoretic origins of the subject, the term definable is also used to refer to them. We will defer to van den Dries [vdD98], a superb guide to the subject, and maintain this logical terminology. A function between definable sets is called definable if its graph is.

Let $\left\langle i_{1}, i_{2}, \ldots, i_{m}\right\rangle$ be a sequence of 0 's and 1 's. The collection of cylindrical $\left\langle i_{1}, i_{2}, \ldots, i_{m}\right\rangle$-cells is defined by induction on $m$. A $\langle 0\rangle$-cell is a singleton in $\mathbb{R}$; a $\langle 1\rangle$ cell is an open (possibly unbounded) interval in $\mathbb{R}$. If $\left\langle i_{1}, i_{2}, \ldots, i_{m-1}\right\rangle$-cells in $\mathbb{R}^{m-1}$ have already been specified, an $\left\langle i_{1}, i_{2}, \ldots, i_{m-1}, 0\right\rangle$-cell is the graph of a continuous, definable, $\mathbb{R}$-valued function on some $\left\langle i_{1}, i_{2}, \ldots, i_{m-1}\right\rangle$-cell $X$. An $\left\langle i_{1}, i_{2}, \ldots, i_{m-1}, 1\right\rangle$ cell is the set of points

$$
\left\{(x, y) \in \mathbb{R}^{m} \mid x \in X, f(x)<y<g(x)\right\},
$$

where $X$ is an $\left\langle i_{1}, i_{2}, \ldots, i_{m-1}\right\rangle$-cell and $f, g$ are continuous, definable $\mathbb{R}$-valued functions on $X$ with $f<g$. (Here $f \equiv-\infty$ or $g \equiv+\infty$ are also permitted.) The dimension of an $\left\langle i_{1}, i_{2}, \ldots, i_{m}\right\rangle$-cell is $\sum_{k=1}^{m} i_{k}$. (For o-minimal structures over $\mathbb{R}$, this is the same as the topological dimension; in the axiomatic setting, this formula serves as a definition.) Note that any cylindrical cell is definable.

Any definable set $X$ possesses finite decompositions into cylindrical cells and one can let

$$
\mathrm{eu}_{\mathcal{S}}(X)=\sum_{\alpha \in \operatorname{cell}(X)}(-1)^{\operatorname{dim}(\alpha)}
$$

for any such decomposition. When $\mathcal{S}$ is the collection of semi-algebraic sets, this definition is consistent with the one involving triangulations into relatively open simplices, as in Knebusch and Delfs [DK82]. We will need the following property of the combinatorial Euler characteristic: if $U$ is a definable subset of the definable set $X$ then $\mathrm{eu}_{\mathcal{S}}(X)=\mathrm{eu}_{\mathcal{S}}(U)+\mathrm{eu}_{\mathcal{S}}(X-U)$. This follows from the fact that the collection of cylindrical cell decompositions of $X$, ordered under refinement, is a filtered poset that is cofinal in the poset of all decompositions of $X$ into definable pieces.

Let $\mathcal{S}^{(1)}, \mathcal{S}^{(2)}$ be o-minimal structures over $\mathbb{R}$; let $A$ be an $\mathcal{S}^{(1)_{-}}$and $B$ an $\mathcal{S}^{(2)}$ definable set. The main result is

Theorem 2.2. If $A$ is homeomorphic to $B$, then $\mathrm{eu}_{\mathcal{S}^{(1)}}(A)=\mathrm{eu}_{\mathcal{S}^{(2)}}(B)$.

Specializing to $\mathcal{S}^{(1)}=\mathcal{S}^{(2)}=$ semi-algebraic sets:

Corollary 2.3. If $A, B$ are affine semi-algebraic sets that are homeomorphic, then $\mathrm{eu}(A)=\mathrm{eu}(B)$. 
In Thm. 2.2 it is not assumed that $\mathcal{S}^{(1)}$ and $\mathcal{S}^{(2)}$ have a common o-minimal extension. Of course, the generality afforded by stating the theorem in the above form is partly a mirage. If $\mathcal{S}^{(1)}$ and $\mathcal{S}^{(2)}$ both extend semi-algebraic sets, then there is an $\mathcal{S}^{(1)}$ _ definable homeomorphism between $A$ and a semi-linear set (i.e., an $\mathcal{S}^{(1)}$-triangulation of $A$ ), and also an $\mathcal{S}^{(2)}$-triangulation of $B$. Since eu $\mathcal{S}_{\mathcal{S}}$ is certainly invariant under $\mathcal{S}$ definable homeomorphisms, under the assumption that $\mathcal{S}^{(1)}$ and $\mathcal{S}^{(2)}$ both extend semi-algebraic sets Thm. 2.2 follows from Cor. 2.3.

A result closely related to Cor. 2.3 was proved by McCrory and Parusinski by entirely different methods, cf. Remark A.7. of [MP97]: Let $h: X \rightarrow X$ be a homeomorphism (not necessarily semi-algebraic) of semi-algebraic sets. Let $\phi \in F(X)$ be such that $\phi^{\prime}=\phi \circ h \in F(X)$. Let $Y \subset X$ be a compact semi-algebraic subset such that $Y=h^{-1}(Y)$ is also semi-algebraic. Then

$$
\int_{Y} \phi=\int_{Y^{\prime}} \phi^{\prime}
$$

(Here $F(X)$ is the ring of semi-algebraically constructible functions and integration is with respect to Euler characteristic as measure.) Taking $Y=X$ and $F$ to be the characteristic function of a semi-algebraic subset of $X$, this means that the combinatorial Euler characteristic of an embedded semi-algebraic set is invariant with respect to homeomorphisms that extend to some compact semi-algebraic neighborhood. The proof by McCrory and Parusinski uses the possibility of expressing any semi-algebraic set as a topologically defined boolean combination of (possibly larger) closed semialgebraic subsets of the ambient space. The argument in this paper stays inside the given set, with the help of a topologically defined stratification of o-minimal sets.

\section{The canonical stratification}

Given a topological space $X$, let us, as it were, try to extract its locally compact 'core' by a 'greedy algorithm'. That is, set

$$
z(X):=\{x \in X \mid x \text { has a compact neighborhood in } X\} .
$$

Lemma 3.1. (i) $z(X)$ is open (possibly empty) in $X$. (ii) If $X$ is Hausdorff, then $z(X)$ is locally compact.

Proof. (i) If $x \in U \subseteq C$ with $U$ open and $C$ compact, then $U \subseteq z(X)$ as well. (ii) For all $y \in C-U$, let $V_{y}, W_{y}$ be disjoint opens in $X$ with $x \in V_{y}, y \in W_{y}$. Since $C-U$ is compact, there is a finite $I \subseteq C-U$ such that $\left\{W_{i} \mid i \in I\right\}$ covers $C-U$. Then

$$
x \in U \cap\left(\bigcap_{i \in I} V_{i}\right) \subseteq C-\left(\bigcup_{i \in I} W_{i}\right) \subseteq U \subseteq z(X) .
$$

But $U \cap\left(\bigcap_{i \in I} V_{i}\right)$ is open and $C-\left(\bigcup_{i \in I} W_{i}\right)$ is compact.

Note that a topological space need not have a maximal locally compact subspace, and $z(X)$ could well be empty (e.g., take $X$ to be the rationals with the metric topology) even though any space has locally compact subsets, namely, the finite ones. Thankfully, $z(-)$ is well-behaved on o-minimal sets over $\mathbb{R}$. 
Definition 3.2. A stratification of a topological space $X$ will mean a finite decomposition

$$
X=\bigsqcup_{\alpha \in I} \alpha
$$

such that the closure $\bar{\alpha}$ of any stratum is a union of strata. A stratum $\alpha$ is maximal if $\alpha \subseteq \bar{\beta}$ with $\beta$ a stratum implies $\alpha=\beta$.

We will not need to place manifold or regularity conditions on stratifications.

Lemma 3.3. (i) Any maximal stratum is open. (ii) If the strata are locally compact, then $z(X)$ contains all maximal strata.

Proof. ( $i$ ) If $\alpha$ is maximal, then it must be the complement of the union of the closures of the other strata. (ii) By (i), a compact neighborhood of $x$ in $\alpha$ is a compact neighborhood of $x$ in $X$.

Let us now work in a fixed o-minimal structure over $\mathbb{R}$. We will freely use the results of chapters 3 and 4 of van den Dries's monograph, and will also follow his convention of setting the dimension of the empty set to be $-\infty$.

Proposition 3.4. If $X$ is a definable set, so is $z(X)$ and $\operatorname{dim}(X-z(X))<\operatorname{dim}(X)$.

Proof. Thanks to locally compact and locally closed being the same for subsets of $\mathbb{R}^{n}, z(X)$ is first-order definable via

$$
\begin{aligned}
& z(X)=\{x \in X \mid \text { there is an } \epsilon>0 \text { such that for all } y \in \bar{B}(x, \epsilon) \\
& \qquad \text { if } y \notin X \text { then there is a } \delta>0 \text { such that } B(y, \delta) \cap X=\varnothing\},
\end{aligned}
$$

where $B(x, r)$ is the open and $\bar{B}(x, r)$ the closed ball of radius $r$ centered at $x$. (Replace 'ball' with 'box' if it is desirable to work over the structure $\langle\mathbb{R},<\rangle$.)

Now any definable set permits a stratification into cylindrical cells. Since those are locally compact, this is precisely the situation of Lemma 3.3. Hence $z(X)$ contains all maximal cells. Let $d=\operatorname{dim}(X)$. Since for any definable set $U$, $\operatorname{dim}(\bar{U}-U)<\operatorname{dim}(U)$, all $d$-dimensional cells in the stratification are maximal. $X-z(X)$ is therefore a definable subset of a union of cells of dimension less than $d$ and $\operatorname{dim}(X-z(X))<$ $\operatorname{dim}(z(X))=\operatorname{dim}(X)$.

Definition 3.5. The canonical decomposition of a definable set $X$ is set up by induction as follows

- $X_{0}:=z(X)$

- for $i>0$,

$$
X_{i}:=z\left(X-\left(X_{0} \cup X_{1} \cup \cdots \cup X_{i-1}\right)\right) .
$$

By Prop. 3.4, each $X_{i}$ is definable and $\operatorname{dim}\left(X_{i}\right)>\operatorname{dim}\left(X_{i+1}\right)$ as long as $X_{i}$ is non-empty. Therefore the iteration terminates and

$$
X=\bigsqcup_{i=0}^{n} X_{i}
$$

for some $n$, with $X_{i} \neq \varnothing$. By Lemma 3.1, each $X_{i}$ is locally compact. 
The canonical decomposition is actually a stratification, and the best bound for $n$ is $\left\lfloor\frac{\operatorname{dim}(X)}{2}\right\rfloor$ rather than the obvious $\operatorname{dim}(X)$. Since these facts are not needed for the proof of the main theorem, we will return to them later.

The next statement is well-known, but let us include it for completeness. Let $K$ be any field and let $H_{c}^{*}(X, K)$ be sheaf cohomology with compact support with constant coefficients $K$. (All spaces will be assumed Hausdorff.) Let

$$
\chi_{c}(X):=\sum_{i=0}^{\operatorname{dim}(X)}(-1)^{i} \operatorname{dim}_{K} H_{c}^{i}(X, K)
$$

be the cohomological Euler characteristic with compact support.

Lemma 3.6. If $X$ is definable and locally compact, then $\mathrm{eu}_{\mathcal{S}}(X)=\chi_{c}(X)$.

This is a consequence of two facts:

(1) For any cylindrical cell $\alpha$, eu $\mathcal{S}(\alpha)=(-1)^{\operatorname{dim} \alpha}=\chi_{c}(\alpha)$ since $\alpha$ is homeomorphic to $(0,1)^{\operatorname{dim} \alpha}$.

(2) If $X=U \sqcup Z$ is an open-closed decomposition of a locally compact space $X$, and if the total cohomology $H_{c}^{*}(U, K)$ as well as $H_{c}^{*}(Z, K)$ are finite-dimensional, then the total cohomology $H_{c}^{*}(X, K)$ is finite-dimensional too; hence the corresponding Euler characteristics are well-defined, and

$$
\chi_{c}(X)=\chi_{c}(U)+\chi_{c}(Z) .
$$

(Cf. Iversen [Ive86] III.7.6.) Given a locally compact definable $X$, fix a stratification of $X$ by cylindrical cells. Now take away from $X$ one maximal (a fortiori, open) cell $U$ at a time and iterate, noting that a closed subset of a locally compact space is locally compact.

Proof of Theorem 2.2. Let $h: A \rightarrow B$ be a homeomorphism. Since the stages of the canonical decomposition are defined purely topologically, $h$ restricts to homeomorphisms $h_{i}: A_{i} \rightarrow B_{i}$ between the stages of $A$ and of $B, i=0,1, \ldots, n$. By Lemma 3.1, 3.6 and the additivity of the combinatorial Euler characteristic

$$
\mathrm{eu}_{\mathcal{S}^{(1)}}(A)=\sum_{i=0}^{n} \mathrm{eu}_{\mathcal{S}^{(1)}}\left(A_{i}\right)=\sum_{i=0}^{n} \chi_{c}\left(A_{i}\right)=\sum_{i=0}^{n} \chi_{c}\left(B_{i}\right)=\sum_{i=0}^{n} \mathrm{eu}_{\mathcal{S}^{(2)}}\left(B_{i}\right)=\mathrm{eu}_{\mathcal{S}^{(2)}}(B) .
$$

Let us return to study some further properties of the canonical decomposition. For strata $\alpha, \beta$ of a stratified topological space $X$, introduce the notation $\alpha \preceq \beta$ for $\alpha \subseteq \bar{\beta}$. This is always a preorder (i.e., $\preceq$ is reflexive and transitive). If $X$ is a definable set stratified into definable subsets, then $\preceq$ is even a partial order, since $\alpha \preceq \beta$ and $\alpha \neq \beta$ will imply $\operatorname{dim}(\alpha)<\operatorname{dim}(\beta)$, so $\preceq$ will be antisymmetric.

Proposition 3.7. The canonical decomposition of any definable set $X$ is a stratification.

Indeed, $z(X)$ is open in $X$ for any space, cf. Lemma 3.1. Assume now $X$ definable and fix a stratification of $X$ by cylindrical cells. By Lemma 3.3, $z(X)$ contains all maximal cells. But any cell is contained in the closure of a maximal one; hence, 
for $X$ definable, $z(X)$ is dense and open in $X$. Iterating, $X_{i}$ is relatively open and dense in $\left(X-\left(X_{0} \cup X_{1} \cup \cdots \cup X_{i-1}\right)\right)$ which is closed in $X$. That is to say, $\overline{X_{i}}=$ $\bigcup_{i \leqslant j \leqslant n} X_{j}$.

Since the canonical stratification is defined by a 'greedy algorithm', it is somewhat natural to guess that it is the coarsest among all stratifications of $X$ with locally compact, definable strata. But that can fail:

Example 3.8. Let $X$ be the subset of the plane $\alpha \cup \beta$ where $\alpha$ is the interior of the triangle with vertices $(0 ; 0),(1 ; 0)$ and $(0 ; 1)$ and $\beta$ is the interval $[0,1]$. Then $z(X)$ is the union of $\alpha$ with the interval $(0,1)$, so it is not a union of existing strata.

This is to be blamed, it turns out, not on the fact that $X$ is not locally compact, but on the fact that the given stratification of $X$ does not extend to $\mathbb{R}^{2}$. The canonical stratification of a definable $X$ is coarsest among those that are part of a stratification of an ambient locally compact definable space. A consequence of this is that for definable sets the dimension between successive strata of the canonical stratification drops by at least 2 ; cf. Cor. 3.12 .

Let $W$ be a stratified topological space. In what follows, lowercase greek letters range over strata of $W$.

Lemma 3.9. Let $Z$ be a union of strata of $W$.

(i) $Z$ is closed in $W$ if and only if for every $\gamma \subseteq Z$, if $\beta \preceq \gamma$ then $\beta \subseteq Z$.

(ii) $Z$ is open in $W$ if and only if for every $\alpha \subseteq Z$, if $\alpha \preceq \beta$ then $\beta \subseteq Z$.

(iii) $Z$ is locally closed in $W$ if and only if for every $\alpha, \gamma \subseteq Z$, if $\alpha \preceq \beta \preceq \gamma$ then $\beta \subseteq Z$.

Proof. (i) is saying that $Z$ is a union of closures of strata. (Note that stratifications are required to be finite.) (ii) says that $Z$ is the complement of a union of closures of strata.

(iii), if: Let $Z_{\downarrow}$ be the union of $\{\beta \subseteq W \mid \beta \preceq \gamma$ for some $\gamma \subseteq Z\}$; let $Z^{\uparrow}$ be the union of $\{\beta \in W \mid \alpha \preceq \beta$ for some $\alpha \in Z\}$. $Z_{\downarrow}$ is closed by (i), $Z^{\uparrow}$ is open by (ii). $Z \subseteq Z_{\downarrow} \cap Z^{\uparrow}$ clearly. $Z \supseteq Z_{\downarrow} \cap Z^{\uparrow}$ by the given condition.

(iii), only if: Suppose $Z=U \cap V$ with $U$ open and $V$ closed, $\alpha, \beta, \gamma$ as assumed. $\beta \subseteq V$ since $\beta \prec \gamma$ and $V$ is closed. $\beta \cap U \neq \varnothing$ since $\alpha \prec \beta$ and $U$ is open. Thence $\beta \cap Z \neq \varnothing$. So $\beta \subseteq Z$ since $Z$ is a union of strata.

The next proposition says that if the stratification of a definable $X$ is part of the stratification of an ambient locally compact definable $W$, then $z(X)$ is the union of the 'top intervals' in the poset of strata of $X$.

Proposition 3.10. Let $W$ be definable, locally compact and stratified into definable sets. Let $X$ be a union of strata. Define

top $(X)=$ union of $\{\alpha \subseteq X \mid$ for all $\beta \subseteq W$ and $\gamma \subseteq X$, if $\alpha \preceq \beta \preceq \gamma$, then $\beta \subseteq X\}$.

Then $z(X)=\operatorname{top}(X)$.

Proof. $z(X) \supseteq$ top $(X)$ : Lemma 3.9(ii) implies that top $(X)$ is open in $X$. (Indeed, let $\alpha \subseteq \operatorname{top}(X), \beta \subseteq X$ with $\alpha \preceq \beta$ and let $\beta \preceq \beta_{1} \preceq \beta_{2}$ with $\beta_{1} \subseteq W, \beta_{2} \subseteq X$. $\beta_{1} \subseteq X$ since $\alpha \subseteq \operatorname{top}(X)$. But that means $\beta \subseteq \operatorname{top}(X)$ by the definition of $\operatorname{top}(X)$.) 
Lemma 3.9(iii) implies that $\operatorname{top}(X)$ is locally closed in $W$. (Indeed, let $\alpha, \gamma \subseteq$ top $(X), \beta \subseteq W, \alpha \preceq \beta \preceq \gamma \cdot \beta \subseteq X$ since $\alpha \subseteq \operatorname{top}(X)$ and then $\beta \subseteq \operatorname{top}(X)$ as before.)

So top $(X)$ is open in $X$ and locally compact. A fortiori every point of $\operatorname{top}(X)$ belongs to $z(X)$.

$z(X) \subseteq \operatorname{top}(X)$ : say $x \in z(X)$ and $x \in \alpha \subseteq X$. Let $\alpha \preceq \beta \preceq \gamma$ be arbitrary with $\beta \subseteq W, \gamma \subseteq X . z(X)$ is locally compact by Lemma 3.1 , hence locally closed in $W$. Write $z(X)=U \cap V$ with $U$ open and $V$ closed in $W$. Let $\gamma_{1}$ be maximal in $X$ and $\gamma \preceq \gamma_{1}$; then $\gamma_{1} \subseteq \operatorname{top}(X) \subseteq z(X) \subseteq V$. So $\beta \subseteq \overline{\gamma_{1}} \subseteq V$. $\beta \cap U \neq \varnothing$ since $U$ is a neighborhood of $x$ and $x \in \bar{\beta}$. So $\beta \cap z(X) \neq \varnothing$. Since $X$ is a union of strata, $\beta \subseteq X$. But this means $\alpha \subseteq \operatorname{top}(X)$.

Corollary 3.11. Let $X \subset \mathbb{R}^{N}$ be definable. Let $X$ be stratified by definable sets in a way that is part of a stratification of all of $\mathbb{R}^{N}$. Then the canonical stratification $X=\bigsqcup_{i=0}^{n} X_{i}$ is coarser than the given one, i.e., each $X_{i}$ is a union of existing strata.

Corollary 3.12. Let $X$ be definable, with canonical stratification $X=\bigsqcup_{i=0}^{n} X_{i}$. Then

$$
\operatorname{dim}\left(X_{i}\right)-\operatorname{dim}\left(X_{i+1}\right) \geqslant 2
$$

for $0 \leqslant i \leqslant n$. (Recall that $X_{n+1}=\varnothing$ and $\operatorname{dim}(\varnothing)=-\infty$ by convention.)

Proof. If $X \subset \mathbb{R}^{N}$, find a stratification of $\mathbb{R}^{N}$ into cylindrical cells that partitions $X$ (cf. van den Dries [vdD98] Ch. 4 Prop. 1.13). Apply Prop. 3.10 with $W=\mathbb{R}^{N}$ and note that if $d=\operatorname{dim}(X)$ then $\operatorname{top}(X)$ contains all cells of dimension $d$ and of $d-1$. Now iterate.

Cor. 3.12 cannot be improved further:

Example 3.13. Let $P_{i}, i=0,1,2, \ldots, 2 n$, be points in general position in $\mathbb{R}^{2 n}$. Let $\operatorname{int}\left\langle P_{0}, P_{1}, \ldots, P_{2 i}\right\rangle$ denote the relative interior of the simplex $\left\langle P_{0}, P_{1}, \ldots, P_{2 i}\right\rangle$ and define

$$
X=\bigcup_{i=0}^{n} \operatorname{int}\left\langle P_{0}, P_{1}, \ldots, P_{2 i}\right\rangle .
$$

Set $W$ to be the closed simplex $\left\langle P_{0}, P_{1}, \ldots, P_{2 n}\right\rangle$ stratified into the relative interiors of all its subsimplices and apply Prop. 3.10. That shows $X_{i}=\operatorname{int}\left\langle P_{0}, P_{1}, \ldots, P_{2(n-i)}\right\rangle$ whence $\operatorname{dim}\left(X_{i}\right)=2(n-i)$.

For general $X$, of course, the strata will not be equidimensional. In fact, it could happen that for all $0 \leqslant i \leqslant n$ and all $0 \leqslant d \leqslant \operatorname{dim}\left(X_{i}\right)$, the stratum $X_{i}$ contains points where the local dimension is $d$.

Closing ruminations. I do not know of a counterexample to eu $(X)=\chi_{c}(X)$, nor to eu being a proper homotopy invariant. For a space that is not locally compact, the textbook theory of cohomology with compact support does not provide an obvious way to determine the cohomology groups. The reason seems to be that the classical repertoire of homological algebra - Mayer-Vietoris sequences, tautness, continuity, Künneth formula, extension by zero and so on - works best for sheaf cohomology with support in a paracompactifying family, and the family of compact subsets of $X$ is paracompactifying if and only if $X$ is Hausdorff and locally compact. Sheaf cohomology with a non-paracompactifying family of supports can indeed be paradoxical; 
for example, the cohomological dimension of $\mathbb{R}^{N}$ is $N+1$ if one allows all families of supports. (See Bredon [Bre97].)

After 'definable', the most frequently occurring phrase in this note must be 'locally compact', but what the arguments turn on is not so much being locally compact as being locally closed in $\mathbb{R}^{N}$; more precisely, the boolean depth of a definable set. For $X \subset \mathbb{R}^{N}$, let $C_{0}=X$ and inductively, $C_{i+1}=\overline{C_{i}}-C_{i}$. For definable $X$, the iteration terminates and writing $Z_{i}=\overline{C_{i}}$, one can express $X$ canonically as a boolean combination

$$
X=Z_{0}-\left(Z_{1}-\left(Z_{2}-\left(\cdots-\left(Z_{k-1}-Z_{k}\right) \cdots\right)\right)\right.
$$

of closed definable subsets of $\mathbb{R}^{N}$, with $Z_{0} \supset Z_{1} \supset \cdots \supset Z_{k} \neq \varnothing . X$ is closed if and only if $k=0$ in this expression, and locally closed (but not closed) if and only if $k=1$. (The space of Ex. 3.8 has boolean depth 2.) For closed, definable subsets $X$ of $\mathbb{R}^{N}$, $\mathrm{eu}(X)=\chi(X)$. The study of cohomology with compact support of a locally compact, Hausdorff space $X$ is basically that of ordinary cohomology of the pair $\left(\check{X}, x_{0}\right)$ where $\check{X}$ is the Cech compactification of $X$ and $x_{0}$ the point at infinity; in our context, this can be substituted by the pair $\left(Z_{0}, Z_{1}\right)$. This suggests that the combinatorial Euler characteristic of a definable set of boolean depth $k$ is a filtered homological invariant constructed from a suitable $k$-ad, which is functorial (and even homotopy invariant) under compatible continuous maps (resp. homotopies), but this class of morphisms gets smaller and smaller as $k$ increases.

\section{References}

[BPR06] Saugata Basu, Richard Pollack, and Marie-Françoise Roy, Algorithms in real algebraic geometry, second ed., Algorithms and Computation in Mathematics, no. 10, Springer-Verlag, 2006.

[Bre97] Glen E. Bredon, Sheaf theory, Graduate Texts in Mathematics, vol. 170, Springer-Verlag, 1997.

[DK82] Hans Delfs and Manfred Knebusch, On the homology of algebraic varieties over real closed fields, J. Reine Angew. Math. 335 (1982), 122-163.

[Ive86] Birger Iversen, Cohomology of sheaves, Universitext, Springer-Verlag, 1986.

[MP97] Clint McCrory and Adam Parusinski, Algebraically constructible functions, Ann. Sci. École Norm. Sup. (4) 30 (1997), no. 4, 527-552.

[Nic10] Liviu I. Nicolaescu, Tame Flows, Memoirs of the American Mathematical Society, no. 980, American Mathematical Society, 2010.

[RSW03] J.-P. Rolin, P. Speissegger, and A. J. Wilkie, Quasianalytic DenjoyCarleman classes and o-minimality, Journal of the American Mathematical Society 16 (2003), no. 4, 751-777.

[Sch91] Stephen Schanuel, Negative sets have Euler characteristic and dimension, Category theory (Como, 1990), Lecture Notes in Mathematics, no. 1488, Springer-Verlag, 1991, pp. 379-385. 
[vdD98] Lou van den Dries, Tame topology and o-minimal structures, London Mathematical Society Lecture Note Series, no. 248, Cambridge University Press, 1998.

Tibor Beke tibor_beke@uml.edu

Department of Mathematics, University of Massachusetts Lowell, One University Avenue, Lowell MA 01854, USA 